$\mathbf{Q}_{-} \mathbf{S}_{\mathrm{crip} t a} \mathbf{R}_{\mathrm{r} \text { manica }}$ vol. 2 (2015): 31-37

\title{
Le recours aux domaines tabouisés dans la publicité sociétale
}

\author{
The use of taboo areas in social advertising
}

\author{
Agnieszka Woch \\ Université de Łódź, Pologne
}

\begin{abstract}
Résumé : L'article traite du tabou dans la publicité sociétale. En s'appuyant sur les exemples relevés dans un corpus de campagnes sociétales francophones, italiennes et polonaises, l'auteur analyse les tabous auxquels recourent les spécialistes de la publicité en question en vue de sensibiliser le destinataire aux sujets importants pour la société contemporaine.
\end{abstract}

Mots-clés : campagne sociétale, tabou, mort, discrimination.

Abstract: This paper discusses the appearance of taboo topics in social advertising. It analyses the taboos employed by the authors of social campaigns in order to raise awareness of social issues relevant to their contemporary society. The corpus comprises slogans from French, Italian and Polish social campaigns.

Keywords: social advertising, taboo, death, discrimination.

La publicité sociétale constitue un type de publicité particulier qui, dans le but de faire passer des messages de prévention de santé, de sécurité routière, de tolérance, etc., a recours aux divers tabous. Elle puise dans les domaines tabouisés non pas pour le goût d'une simple provocation, mais au service de grandes causes. Les campagnes abordant des sujets qui transgressent les interdits ou les superstitions morales et pudiques des sociétés, sont susceptibles de provoquer des controverses et de susciter des réactions et des critiques violentes. Mais les spécialistes en marketing social continuent à impressionner et à choquer le destinataire du message en vue d'attirer son attention et changer des opinions et des attitudes.

Dans les sociétés contemporaines, y compris celles polonaise, française et italienne, il existe toujours un nombre important de sujets que l'on n'aborde pas ouvertement dans une conversation. Par contre, la publicité de sensibilisation les exploite abondamment. Ainsi, dans le corpus des campagnes sociétales que nous avons recueilli, il est possible de relever des sujets tabous, tels que la mort, la maladie (surtout le $\mathrm{VIH}$, le sida, le cancer), le sexe, les discriminations (des malades, des étrangers, des personnes handicapées, des personnes âgées, etc.) et la violence domestique. 


\section{Le tabou de la mort et de la maladie dans la publicité sociétale}

La figure de la mort dans la publicité sociétale fait appel à la peur et elle passe un message difficile à oublier. Elle nous rappelle qu'il est indispensable d' "I adopter un comportement sain au risque de perdre sa vie, que ce soit à cause d'un accident de voiture, un meurtre, un suicide ou le fait de fumer, par exemple " (Dupont, 2013: 6). Les publicitaires exploitent la mort avant tout dans les campagnes de la sécurité routière et dans les campagnes de prévention de la santé (il s'agit surtout du sida et du tabagisme).

Le tabou en question peut être brisé à travers le recours au champ lexical de la mort. C'est le cas des publicités sociétales qui utilisent, entre autres, les substantifs la mort, un mort, comme dans la campagne contre le tabagisme: Fumer peut entraîner une mort lente et douloureuse ou celle de la sécurité routière : 1 verre 2 verres 3 morts. Dans le corpus on peut souvent relever les verbes qui renvoient directement à la mort. Le verbe le plus exploité est le verbe tuer, que l'on utilise pour rappeler au destinataire du message que certaines maladies (le sida, l'hépatite) ou comportements à risque (le sexe non protégé, l'excès de vitesse, l'abus des stimulants) entraînent la mort : En France l'hépatite tue plus que les accidents de la route; Rien ne serait arrivé ... S'ils avaient utilisé un préservatif... Le sida tue, pensezy! ; Prędkość zabija. Włącz myślenie (La vitesse tue. Allume ton cerveau) ; AIDS. Se lo conosci lo eviti, se lo conosci non ti uccide (SIDA. Si tu le connais, tu l'évites, si tu le connais, il ne te tue pas). Les publicitaires ont également le recours au verbe mourir : Je suis venu, j'ai bu et je mourus, parfois aussi à son euphémisme Chi si droga si spegne (Celui qui se drogue, s'éteint) et au verbe empoisonner, dans les cas des campagnes contre le tabagisme: Chi fuma avvelena anche te. Digli di smettere (Celui qui fume, t'empoisonne. Dis-lui d'arrêter). L'imminence de la mort peut être aussi suggérée par le choix d'une expression qui l'évoque. C'est le cas de la campagne de la sécurité routière qui exploite l'expression populaire sentir le sapin (être près de la mort). Le texte Ça sent le sapin est inscrit sur un "sapin magique " odorant l'intérieur de la voiture; il attire ainsi l'attention sur les accidents mortels provoqués par les jeunes conducteurs. Les publicitaires recourant au champ lexical de la mort exploitent également des adjectifs tels que mort, mortel ou meurtrier pour désigner soit la cause, soit la conséquence d'un comportement risqué, comme dans les exemples suivants: Vous avez juste oublié un clignotant, il est juste un peu mort; Dès $20 \mathrm{~km} / \mathrm{h}$, un choc sans ceinture peut être mortel ; La route la plus familière est souvent plus meurtrière.

La mort ne doit toujours pas être présente au niveau verbal. Elle apparaît aussi en images où on l'évoque au travers des symboles tels que le sang, une croix, un enterrement. La mort peut être présentée comme conséquence d'un accident causé par l'excès de vitesse, de la drogue ou d'alcool mais aussi par le biais d'une maladie telle que le sida, I'IST, la tumeur. En recourant au tabou de la mort d'une façon implicite, les campagnes sociétales rappellent la cause ou montrent des conséquences des actions entreprises par les hommes. C'est le cas de la campagne de la sécurité routière qui a recours à deux affiches. Sur le premier on voit un siège, dans le cas échéant en brique, sur le deuxième, un siège en avant avec une tomate écrasée, qui fait penser à un cerveau après un accident, et les mots Attachez votre ceinture à l'arrière. 
Un autre symbole fréquent est le verre d'alcool. C'est le cas de la campagne 1 verre, 2 verres, 3 MOR+S où la lettre † est remplacée par une croix blanche. Les caractères du dernier élément de l'énumération évoquée sont en rouge et font penser à la couleur du sang. Le sang est l'un des symboles auquel la sécurité routière recourt abondamment en l'évoquant au travers de la couleur des caractères ou des gouttes, des taches rouges sur la route ou bien des images des corps blessés. Tel est le cas de la campagne Ta dernière ligne blanche dont l'affiche montre une ligne de signalisation horizontale tachée de sang. Dans les cas de la campagne En ville il n'y a pas que des voitures accidentées par la recherche de la bienséance on n'a représenté qu'un torse et une jambe cassée qui appartiennent aux personnes qui semblent en chair et en os mais dont le corps est réalisé en plastique. Le texte L'oubli du clignotant fait chaque jour 27 nouvelles victimes / Chaque jour, 15 personnes sont victimes du non-respect $d u$ feu explique l'image. En ce qui concerne les conséquences funestes d'un comportement à risque, les publicitaires suggèrent la mort sur des affiches où on relève des véhicules cassés (Dès 2 verres, le risque existe), les pieds d'un décédé (Levez le pied. $163 \mathrm{~km} / \mathrm{h}$ ), un sac en plastique (Nie zasuwam / Je ne roule pas vite) ou bien des symboles qui font penser à un enterrement tels que : la couronne meurtrière (Sur la route, changeons notre comportement avant qu'il ne soit trop tard), un cercueil à la place de la tête d'un conducteur au volant (Gueule de bois ce week-end? Chaque week-end, 4 tués sur nos routes) l'image d'une fille morte les fleurs dans ses mains (Rien ne serait arrivé ... s'ils avaient utilisé un préservatif), une couronne mortuaire dans la publicité contre le sida (HIV nie wybiera. Ty możesz / VIH ne choisit pas. Tu peux) ou le cimetière (Parking payant, places disponibles au sous-sol).

\section{Le tabou des discriminations}

La publicité sociétale vise aussi des tabous de la discrimination fondée sur la religion, la race ou l'orientation sexuelle en créant des campagnes de sensibilisation assez fortes. La première que nous souhaitons citer est une campagne italienne dont le but est d'informer les victimes et les témoins des discriminations fondées sur des clichés tels que la race, l'âge, la religion, l'infirmité, l'orientation sexuelle où recourir pour les dénoncer. Sur l'affiche de la campagne on relève quatre visages et le slogan Spegni le discriminazioni accendi i diritti (Éteins les discriminations, allume les droits). Un autre exemple, cette fois-ci polonais, est la campagne polonaise Jedni z wielu (Les uns parmi tant d'autres) qui évoque le stéréotype des Roms. Le but de la campagne est de démontrer qu'il y en a plusieurs qui ont réussi dans la vie et que l'origine ne leur empêche pas d'être de bons citoyens ou de faire une carrière. Deux femmes (une institutrice et une doctorante) et deux hommes (un champion du monde et un mineur) ont parlé de leur vie dans les spots et ont prêté leurs visages à la campagne dont les slogans sont les suivants: Jest Polkq. Jest Romka. Jest nauczycielka. Ewelina. Jedna z wielu (Elle est polonaise. Elle est rom. Elle est institutrice. Ewelina. Une parmi tant d'autres), Jest Polkq. Jest Romka. Jest doktorantka. Agnieszka. Jedna z wielu (Elle est polonaise. Elle est rom. Elle est doctorante. Agnieszka. Une parmi tant d'autres), Jest Polakiem. Jest Romem. Jest mistrzem świata. Gérard. Jeden z wielu (II est polonais. II est rom. II est champion du monde. Gérard. Un parmi tant d'autres) ; Jest Polakiem. Jest Romem. Jest górnikiem. 
Rafal. Jeden z wielu (II est polonais. II est rom. II est mineur. Rafał. Un parmi tant d'autres).

La campagne italienne qui lutte contre la discrimination fondée sur l'orientation sexuelle constitue un troisième exemple du tabou des discriminations dans la publicité sociétale. Sur l'affiche on voit trois personnes avec des étiquettes au-dessus de leurs têtes: Alto. Lesbica. Rosso (Grand. Lesbienne. Roux) ce qui est complété par I'information au-dessous : $E$ non c'è altro da dire. Si alle differenze. No all'omofobia (II n'y a rien d'autre à dire. Oui aux différences. Non à l'homophobie).

Parfois le tabou de la maladie, de la discrimination et du sexe se rejoignent dans les cas des campagnes qui luttent pour les droits des personnes malades menacées soit d'une compassion pas attendue de la part de la société soit d'un rejet. Les campagnes luttent contre les préjugées concernant les personnes touchées par le cancer ou le $\mathrm{VIH}$, contre le manque de conscience sur les voies de transmission du virus, contre la peur irrationnelle de la société due aux informations incomplètes, tout en promouvant le droit des personnes malades à la dignité, au respect, à l'amour et à la vie sexuelle. Une campagne audacieuse a été lancée par l'Institut National du Cancer et par le Ministère Français de la Santé. Son slogan : Je suis une personne, pas un cancer a pour but de changer, d'un côté, le regard des Français sur les cancers et, d'un autre côté, ceux des personnes malades. Elle prouve qu'en étant moins distants et moins fatalistes, il est possible de diminuer les conséquences de la maladie sur la vie sociale des personnes touchées. La campagne attire l'attention sur le progrès de la médecine qui permet de traiter un nombre toujours plus important de cancers et explique qu'il n'existe pas de raisons pour mettre à l'écart de la société les malades et de les traiter comme des " cas » inguérissables.

Nous pourrions citer aussi la campagne de la fondation Rak N'Roll visant le tabou de la maladie et du sexe. Les initiateurs l'ont intitulée KancerSutra (CancerSoutra) et son slogan Jeśli rak zabrał część ciebie, cala resztę wypełnij radościa (Si le cancer a pris une partie de toi, remplie de joie tout le reste) incite les personnes touchées de se réjouir de leur vie sexuelle pour améliorer la qualité de leur vie.

Il en est de même dans le cas de la campagne polonaise lancée en juillet 2015 HIVokryzja. Wyleczmy się (VIHocrisie. Soignons-nous). Le mot valise créé pour les besoins de la campagne désigne, comme le communiquent ses auteurs, une hypocrisie envers les personnes séropositifs, apparemment acceptées, mais seulement jusqu'au moment où on apprend qu'elles se trouvent dans l'entourage. À ce moment-là, comme l'explique Kasia, une fille qui vit avec VIH et qui est le visage de la campagne sociétale, il s'avère que les gens ne veulent plus travailler avec une personne séropositive, commencent à avoir peur d'utiliser les mêmes couverts et de lui serrer la main. La campagne brise le tabou de la maladie en y parlant et en éduquant les personnes tout en soulignant que le vrai problème n'est pas celui du virus, qui peut être contrôlé à l'aide des médicaments, mais celui de l'ostracisme de la part de la société. Une autre campagne de succès Si j'étais séropositif a été lancée en France et reprise ensuite au Québec, au Maroc et en Italie (ici à l'occasion de la campagne électorale en 2013). Elle a exposé le portrait des personnalités du monde de la musique, du sport et de la télévision qui posent des questions au grand public pour souligner l'absurdité de la discrimination et pour faire réfléchir les destinataires du message sur les situations de rejet et de stigmatisation 
(www.aides.org). À titre d'exemple: Est-ce que vous aimeriez autant ma gueule si j'étais séropositif ? (Johnny Hallyday), Est-ce que je serais encore au 20 heures si j'étais séropositive? (Claire Chazal), Est-ce qu'on m'engagerait dans les meilleurs clubs d'Europe si j'étais séropositif ? (Didier Drogba), Est-ce que vous me trouveriez aussi drôle si j'étais séropositive ? (Muriel Robin), Seriez-vous prêt à tout essayer avec moi si j'étais séropositif ? (Laurent Ruquier) et ainsi de suite. Toutes les questions ont été accompagnées du slogan de la campagne, à savoir, C'est le sida qu'il faut exclure, pas les séropositifs.

\section{Le tabou et le sexe}

Les campagnes sociétales parlent du sexe dans un but éducateur. II s'agit plutôt de bons réflexes à adopter pour éviter de contracter une maladie. Ce n'est pas donc le tabou de la nudité qui est exploité. II s'agit surtout de promouvoir l'utilisation du préservatif et le dépistage. Les campagnes rappellent que la maladie n'exclut personne et elles brisent le tabou du sexe. En y parlant explicitement et en faisant appel à la raison, elles invitent à la réflexion et incitent aux rapports sexuels protégés, comme dans le cas de la campagne suivante: Ce soir tu risques de coucher avec: Zoé, qui sortait avec Sam, qui a fait l'amour avec Inès, qui a fait une fellation à Damien, qui a forniqué avec Arthur, qui a eu des rapports avec Fred, qui a serré Selma, qui s'est tapé Élodie, qui a eu une aventure avec Jean qui ... PROTÉGEZVOUS!

Une autre manière d'aborder le sujet pour faire passer un message important est de plaisanter, de prendre un ton léger grâce à une rime ou bien aux images drôles. C'est ainsi dans la campagne dont le slogan est Sans Capote, t'es kaput et de la campagne sur l'affiche de laquelle deux lapins se parlent de la manière suivante: Gardons le réflexe ... Adoptons le latex. Une autre publicité exploite le visage d'une vieille dame élégante à côté duquel se trouve le message : La capote protège du sida. Odette. 13874 capotes. Une autre campagne s'appuie sur une image drôle d'un sumo vêtu en tutu, accompagnée d'un texte éducateur : Une IST, ce n'est pas l'inimitable sumo en tutu. Une IST, c'est une Infection Sexuellement Transmissible.

Le tabou lié aux relations sexuelles est également exploité dans les campagnes contre le tourisme sexuel et contre la prostitution, surtout celle des mineurs. C'est le cas de la campagne sociétale où on a recours à l'image d'un homme nu dans le lit qui se reflète dans le miroir. Sous le miroir on voit un sac à dos et les chaussures appartenant à une jeune fille: En France des milliers d'enfants sont prostitués. II est temps d'agir mentionne le texte au-dessous. Dans une autre campagne on voit une fille dans la rue et une voiture. Le texte de la conversation Combien? Trois ans fermes rappelle aux adultes que les relations sexuelles avec les mineurs sont illégales. II y en est de même dans la campagne italienne Potrebbe essere tua figlia (Elle pourrait être ta fille) où on aperçoit la silhovette d'une jeune fille qui s'appuie sur une voiture et parle à son conducteur pour négocier le prix.

\section{Le tabou de la violence domestique}

En ce qui concerne la violence, les messages de la publicité sociétale brisent directement le tabou, ils appellent les violences par leur nom, ils les expliquent : Les 
violences faites aux femmes c'est lorsqu'il: terrorise, insulte, méprise, fait du chantage, menace, hurle, étrangle, viole, prive de liberté, tue. Ils ont recours aux mots désignant les actes de violences et ils incitent à en parler : Parler des violences c'est déjà les combattre. Viols, Forcer, Abus de Pouvoir, Mariage forcé, Hurlements, Sexismes, Humiliation, Maltraitance, Coups, Menaces, etc. Dans la majorité des cas, les messages combattant la violence fournissent aux victimes ou aux témoins une aide, les messages contiennent un numéro que l'on peut appeler: En France une femme meurt tous les jours sous les coups de son mari ou compagnon. Parlez-en avant de ne plus pouvoir le faire. Appelez le 3919. Les plus souvent les auteurs des campagnes ont recours aux verbes à l'impératif: Non à la violence faite aux femmes. Collègues, voisins, amis... Réagissons! Appelez-le 3919 ; Reaguj na przemoc (Réagis contre les actes de violence), Zobacz, usłysz, pomóż (Regarde, entends, réagis !). Sur les affiches on relève les victimes de la violence, les femmes avec des bleus, les femmes qui crient, les enfants qui pleurent et parfois même du sang et les corps des personnes décédées.

\section{Conclusions}

La publicité sociétale n'évite pas les sujets difficiles, elle emploie les termes et les images que la bienséance ou la peur ont rendu tabou. Même si elle puise dans de divers domaines tabouisés, elle exploite avant tout la figure de la mort. Cela a lieu dans les campagnes de la sécurité routière, de prévention de la santé mais aussi dans les campagnes contre l'exclusion sociale ou contre la violence. Les publicitaires rappellent au destinataire du message que les comportements à risque, tels que rouler trop vite, conduire après avoir bu ou en utilisant un smartphone au volant ou bien avoir des relations sexuelles non protégées, peuvent s'avérer fatals. Le tabou de la mort est visible dans les campagnes qui recourent également aux tabous du sexe (ici non protégé ou avec des mineurs), de la violence ou de la discrimination.

Le plus souvent les campagnes brisent le tabou d'une façon audacieuse : on en parle directement, on montre les images fortes et choquantes (des cadavres, des victimes battues, des jeunes qui se prostituent) ou on suggère l'imminence de la mort par des symboles (le cercueil, la couronne mortuaire, le cimetière, le sang). Parfois, surtout dans les campagnes incitant à éviter des IST, on relève le recours à l'humour. On plaisante pour faire passer un message important sans le ton moralisateur, le virus est présenté comme un sumo en tutu, les lapins parlent de l'usage du latex, un préservatif souriant rappelle au destinataire qu'il est kaput sans capote. La technique de l'humour permet de sensibiliser le public en faisant sourire et permet de traiter un sujet sérieux d'une façon atténuée (cf. Woch, 2014).

Dans la publicité sociétale le tabou linguistique est presque absent. Les euphémismes sont très rares, on privilégie un style direct, des mots explicites et forts ou le langage non standard. Le champ lexical de la mort et son image prédominent et deviennent une chose fréquente et très explicite dans les campagnes sociétales polonaises, francophones et italiennes qui font partie de notre corpus. Pourtant, il existe le risque que le destinataire, à force d'être constamment exposé aux termes et aux images violentes, finisse par se désensibiliser. Cela surement aura une influence sur les publicitaires qui devront penser à changer leur stratégie pour attirer 
l'attention, faire appel à la peur et choquer le destinataire des campagnes sociétales.

\section{Bibliographie}

COSSETTE, C. et P. DAIGNAULT (2011) : La publicité sociale : définitions, particularités, usages. Québec : Télémaque.

DĄBROWSKA, A. (2008): "Zmiany obszarów podlegajacych tabu we współczesnej kulturze". Język a kultura, tom 20, Wrocław, pp. 173-196.

DUPONT, L. (2013) : "Esquisse d'une sémiologie de l'image au service des grandes causes en publicité sociale $\%$. Revue française des sciences de l'information et de la communication, 3, pp. 1-11.

LESZCZYŃSKI, Z. (1988): Szkice o tabu językowym. Lublin: KUL.

WOCH, A. (2014) : "Les émotions et les procédés esthétiques dans la publicité sociétale ॥. e-Scripta Romanica, vol. 1, pp. 9-15. 